\title{
Designing to Support Situation Awareness across Distances: An Example from a Scientific Collaboratory
}

\author{
Diane H. Sonnenwald* \\ Kelly L. Maglaughlin** \\ Mary C. Whitton** \\ *University of Borås, Sweden \\ **University of North Carolina, USA \\ Corresponding Addresses \\ Diane H. Sonnenwald \\ Before August, 2003: \\ CB \#3360 \\ University of North Carolina \\ Chapel Hill, NC 27599-3360 \\ Phone: 919-962-8065 \\ E-mail: sonnenwald@ils.unc.edu \\ After August, 2003: \\ University of Borås \\ SE-501 90 Borås \\ Sweden \\ E-mail: Diane.Sonnenwald@hb.se \\ Kelly L. Maglaughlin \\ CB \#3360 \\ University of North Carolina \\ Chapel Hill, NC 27599-3360 \\ E-mail: maglk@ils.unc.edu \\ Mary C. Whitton \\ CB \#3175 \\ University of North Carolina \\ Chapel Hill, NC 27599-3175 \\ E-mail:whitton@cs.unc.edu
}

- Please send all correspondence to Diane Sonnenwald. 


\section{Abstract}

When collaborating individuals rely on situation awareness (the gathering, incorporation and utilization of environmental information) to help them combine their unique knowledge and skills and achieve their goals. When collaborating across distances, situation awareness is mediated by technology. There are few guidelines to help system analysts design systems or applications that support the creation and maintenance of situation awareness for teams or groups. We propose a framework to guide design decisions to enhance computer-mediated situation awareness during scientific research collaboration. The foundation for this framework is previous research in situation awareness and virtual reality, combined with our analysis of interviews and observations of collaborating scientists. The framework suggests that situation awareness is comprised of contextual, task and process, and socio-emotional information. Research in virtual reality systems suggests control, sensory, distraction and realism attributes of technology contribute to a sense of presence (Witmer \& Singer, 1998). We suggest that consideration of these attributes with respect to contextual, task and process, and socio-emotional information provides insights to guide design decisions. We used the framework when designing a scientific collaboratory system. Results from a controlled experimental evaluation of the collaboratory system help illustrate the framework's utility.

Keywords: situation awareness, collaboration, collaboratory, systems design 


\section{Designing to Support Situation Awareness across Distances: An Example from a Scientific Collaboratory}

\section{Introduction}

Collaboration has emerged as a fundamental component of the scientific research process. Through collaboration, scientists can bring their unique knowledge and multiple resources to bear on complex problems, making innovative discoveries that an individual scientist working independently may not. An important component of collaboration is situation awareness. Situation awareness has been linked to performance outcomes in many domains (Endsley, 2000). Interviews with and observations of collaborating scientists reveal that they rely heavily on situation awareness for scientific research activities such as: coordinating complex tasks, establishing mutual confidence, drawing attention to scientific details, developing a working understanding of new concepts, and synthesizing results into new knowledge. Scientists we interviewed explained:

Body language...seeing a person as you talk...helps the flow of conversation ... When we talk with each other...we use our hands to kind of imitate the sample or what just happened.

[Sample preparation is] not so well written up in papers...often there are little tricks that people don't write up in papers. [The tricks] are somewhat crucial but they sound almost stupid if you mention them ... [others can] never make it work unless [they] watch you do it.

Today, information and communications technology has the potential to facilitate collaboration among geographically distributed individuals. However, when collaborators are not co-located, many of the physical cues that help create and maintain situation awareness are absent. For example, hand motions that a remote colleague is using to help explain phenomena may be omitted from the video camera view or not transmitted with sufficient fidelity and speed to be useful. The collaborators are totally dependent on technology to deliver and provide access to information necessary for them to create and maintain situation awareness across distances.

There are few guidelines to help system analysts design systems and/or applications that support the creation, maintenance and use of situation awareness for teams or groups. In fact, the concept, situation awareness, is variously defined and used in the literature. We examined and synthesized the literature, and conducted a field study focusing on collaborative science to create a framework to guide design decisions and system requirements, or specifications, that will enhance awareness. The framework proposes that technology should support the acquisition and dissemination of contextual, task and process, and socio-emotional information. Furthermore, control, sensory, distraction and realism attributes of technology should be examined with respect to supporting the acquisition and dissemination of these types of information. We applied the framework to design the nanoManipulator collaboratory, a system that allows pairs of scientists to see, feel and modify biological samples at the nanometer scale with an atomic force 
microscope across distances. An experimental evaluation of the collaboratory system illustrates the potential utility of the framework.

\title{
Situation Awareness Theory
}

Situation awareness has been defined as:

\begin{abstract}
Continuous extraction of environmental information, integration of this information with previous knowledge to form a coherent mental picture in directing further perception and anticipating future events. (Vidulich, Dominquez, Vogel, \& McMillan, 1994, p. 11)
\end{abstract}

It is a general sense of knowing about things that are happening in the immediate environment and includes having both an accurate understanding of the situation and the knowledge to respond appropriately as the situation evolves. In addition to the assumption that knowledge is a direct reflection of the information we gather from the physical world, knowledge is understood to be an interpretation, based often on idiosyncratic schemas, of the information we gather from the physical world. An individual's pre-existing knowledge and cognitive processing skills influence her situation awareness (Salas, Prince, Baker and Shresta, 1995). Situation awareness is the continuous extraction, integration and use of environmental knowledge by a single person such as a military pilot (Dominguez, 1994). However, situation awareness is not limited to high stress activities like flying fighter jets. Common tasks such as driving a car, walking through a building or participating in a meeting also require situation awareness (Endsley, 1995). Regardless of the situation, individuals "must do more than simply perceive the state of their environment. They must understand the integrated meaning of what they are perceiving in light of their goals before they can choose a suitable action" (Endsley, 1995, pp.33-34). Thus, the theory of situation awareness "integrates individual and social levels of cognitive orientation" (Cool, 2001, p. 25.)

The concept of situation awareness has also been extended to teams ${ }^{1}$. Team, or group, situation awareness has been defined as "an understanding of the activities of others, which provides a context for your own activity" (Dourish \& Bellotti, 1992, p. 107). The team's goals, needs and resources motivate the creation and maintenance of a shared situation awareness among team members. Due to the complexity of most team situations, no one individual can develop and maintain a situation awareness that covers all knowledge required for a given teamwork situation. Not all team members can, or should, have the same shared understanding. Ideally, teams develop an interwoven situation awareness, that is, sufficient overlap among individual team member's awareness so that all members are interconnected in some way, forming a woven tapestry of awareness (Sonnenwald \& Pierce, 2000.) Team situation awareness is facilitated by team processes or behaviors that allow shared knowledge to be developed and maintained (Salas, Prince, Baker, \& Shresta, 1995.) Examples of such behaviors include multiple and redundant communication channels, implicit cues, and spatial

\footnotetext{
${ }^{1}$ Teams are two or more individuals who interact to achieve a common goal and have specific roles or functions to perform when working toward the team's goal (Salas, Dickinson, Converse, \& Tannenbaum, 1992).
} 
co-references that enable individuals to understand the meaning of each other's utterances (Clark, 1996).

The concept of team situation awareness is related to the concept of workspace awareness introduced by Gutwin and Greenberg (1997, 1998, 1999, 2000). Workspace, or groupware, applications are computer applications that allow team members to create, share and manipulate digital artifacts used when performing tasks to achieve their common goal. Workspace awareness is "awareness of people and how they interact with the workspace [application]" (Gutwin \& Greenberg, 2002, p. 417). Workspace awareness involves awareness of the domain tasks and collaboration tasks people are performing when using the workspace application, and to a lesser extent, it also includes awareness of how they are doing these tasks.

A challenge in designing collaboratories and other information systems to support situation awareness across distances is that situation awareness is broadly interpreted (Rodden, 1996; Schmidt, 2002), and lacks specificity (Cool, 2001.) A review of 105 articles in information systems, including computer supported cooperative work, reveals over thirty adjectives used in conjunction with the term "awareness." Examples of these adjectives include: activity, background, casual, contextual, remote, everyday, expanded, gaze, general, informal, interpersonal, mobile, mutual, organizational, participant, passive, peripheral, project, shared and social. These adjectives highlight subtle characteristics of situation awareness. They illustrate that situation awareness can be acquired actively or passively. It is critical for both individual and team performance. It encompasses both individually- and socially-held knowledge. Different types of information as well as information gathering and processing techniques can be used to develop and maintain situation awareness.

Various information systems and tools have been developed, and continue to be developed, in an attempt to support situation awareness. The types of tools include: shared repositories, monitors, audio/video communication, application sharing and virtual environments/reality. Shared repositories, or shared workspaces, are data stores that allow group members to view and update shared work objects, such as scientific data, research notes and documents (e.g., Fowler, Baker, Dargahi, Kouramajian, Gilson, Long, Petermann, \& Gorry, 1994; Kovalainen, Robinson, \& Auramaki, 1998). Monitoring systems provide information about where group members are currently located or what they are currently doing (e.g., Cheverest, Mitchell, Davies, \& Smith, 2000; Cohen, Jacovi, Maarek, \& Soroka, 2000). Audio/video conference systems convey image and/or sound information among remote locations (Dourish \& Bly, 1992; Fish, Kraut, Root, \& Rice, 1992). Shared applications allow remote team members to work together synchronously on an object such as an electronic document, electronic white board, concept map or database (e.g., Roseman \& Greenberg, 1996). Collaborative virtual environments (CVEs), such as multi-user dungeons (MUDs) and MUD object oriented system (MOOs), offer virtual spaces in which team members are represented by avators (graphical icons) and can communicate synchronously, often through text-based applications, e.g., instant messaging applications (e.g., Churchill \& Bly, 1999). These types of tools are neither exhaustive nor mutually exclusive and often one system may have components from more than one category, such as ConferenceXP 
developed by Microsoft, that provides video conferencing and shared applications to collaborators at multiple locations.

However, no one system or set of tools universally facilitates situation awareness. We do not know which characteristics or aspects of situation awareness are most important across contexts and situations, or how those characteristics can be best supported by technology. As Endsley (1995) explains, "Although the pilot and power plant operator each relies on situation awareness, it simply is not realistic or appropriate to expect the same elements to be relevant to both" (p. 37). Although theory currently cannot address this issue, analyses can be conducted to determine which technology and technical features can best support situation awareness in particular contexts and situations. To facilitate such analysis, we propose a framework to guide design decisions with respect to technology to support situation awareness.

\section{Research Approach}

This research was part of a larger study that consisted of two phases. During the first phase, we conducted an ethnographic, or field, study of collaborative work practices in a scientific research setting to develop an understanding of situation awareness and its role in scientific collaboration. The outcomes of this phase included the design framework presented in this paper and requirements for a collaboratory system. During the second phase, we conducted a repeated-measures controlled experiment to compare the outcomes and process of scientific work completed by study participants, working face-to-face and remotely, using the collaboratory system. The research setting for both phases was the nanoManipulator collaboratory.

\section{Research Setting: The nanoManipulator Collaboratory}

Scientific collaboratories are organizational entities that span geographical boundaries using information and communications technology to provide access to scientists, data and/or scientific instruments (e.g., Wulf, 1989, 1993; Finholt, 2001). The goal of the nanoManipulator collaboratory is to provide remote, collaborative access to a specialized scientific instrument, called a nanoManipulator (nM), and to support small groups of scientists as they conduct research that utilizes the $\mathrm{nM}$ instrument. The single-user $\mathrm{nM}$ provides haptic and 3D visualization interfaces to a local (co-located) atomic force microscope (AFM), providing a natural scientist with the ability to interact directly with physical samples ranging in size from DNA to single cells (Finch, Chi, Taylor II, Falvo, et al., 1995; Taylor \& Superfine, 1999).

Hardware elements in the collaboratory system include two PCs. One PC is equipped with a Sensable Devices Phantom ${ }^{\mathrm{TM}}$ force-feedback device. This PC and its associated software provide haptic and 3D visualization interfaces to a local or remote atomic force microscope (AFM) and support collaborative manipulation and exploration of scientific data. Scientists can dynamically switch between working together in shared mode and working independently in private mode. In shared mode, remote, i.e., non-collocated, collaborators view and analyze the same (scientific) data. Mutual awareness is supported via multiple 
pointers, each showing the focus of attention and interaction state for one collaborator. Collaborators can perform almost all operations synchronously. Because of the risk of damage to an AFM, control of the microscope tip is explicitly passed between collaborators. In private mode, each collaborator can independently analyze the same or different data from stream files previously generated. When switching back to private from shared mode, collaborators return to the exact data they were previously using.

Another PC supports shared application functionality and video conferencing (via Microsoft NetMeeting ${ }^{\mathrm{TM}}$ ) and an electronic writing/drawing tablet. This PC allows collaborators to work together synchronously using a variety of domain-specific and off-theshelf applications, including specialized data analysis, word processing and whiteboard applications. Video conferencing is supported by two cameras. One camera is mounted on a gooseneck stand so it can be pointed at the scientist's hands, sketches, or other physical artifacts scientists may use during experiments; the other is positioned to primarily capture a head and shoulders view of the user. Collaborators have software control of which camera view is broadcast from their site. A wireless telephone headset and speakerphone connected to a commercial telephone network provides high quality audio communications for collaborators.

\section{Ethnographic Study}

To develop an understanding of scientific collaborative work practices we used ethnographic techniques (Bloomberg, Giacomi, Mosher, \& Swenton-Hall, 1993; Bloomberg, Suchman \& Trigg, 1996; Huges, Randall \& Shapiro, 1992; Suchman, 1995) including semistructured interviews, critical incident interviews and participant observation. A total of 27 interviews with faculty, post-doctoral and graduate student scientists were conducted. The average length of these interviews was $1.5 \mathrm{hrs}$, with a minimum duration of an hour and a maximum duration of 2.75 hours. All study participants interviewed were actively collaborating with other scientists; some participants were collaborating with each other.

We also observed the scientists on nine occasions as they conducted experiments while working alone and with others. Two types of collaborative interactions were observed: two or more local scientists working together, and a local scientist working with a visiting scientist. Collaboration with visiting scientists was formalized in this setting in that part of one scientist's job was to work with visiting scientists who came to use scientific instruments in the laboratory. We observed two collaborations involving visiting scientists. In one instance, the scientists (postdoctoral researcher and Ph.D. student) had met previously at a conference but had not worked together. In the other instance, the scientists (postdoctoral researcher and industrial scientists) had not previously met in person. In both instances, the visiting scientists had not used the scientific instrument before. Interviews were conducted with the local scientist before these visits, and with all participating scientists after the visits. The interviews and observations were iterative, thereby illuminating discrepancies between perceived and actual work practices (Bloomberg, Giacomi, Mosher, \& Swenton-Hall, 1993; Murray, 1993; Kensing, Simonsen, \& Bodker, 1998). 
We analyzed the data using open and axial coding (Berg, 1989) to discover patterns of work practices. From these patterns, scenarios of work were developed and shared with study participants to refine and verify our understanding of their work practices which included coordinating complex tasks, establishing confidence in each other's scientific skill and knowledge, focusing attention on scientific details, developing a working understanding, and creating new knowledge (Sonnenwald, Bergquist, Maglaughlin, Soo, \& Whitton, 2001).

Based on this understanding and situation awareness theory, we identified three types of knowledge needed by scientists to develop situation awareness. Synthesizing this with research in virtual reality led to design requirements for a collaboratory system. Although many of these requirements were implemented, as typically happens, trade-offs among design features were necessary due to technical, financial and organizational constraints. After the collaboratory system was implemented, we conducted a controlled experiment to evaluate the system.

\section{Controlled Experiment Evaluation}

The experimental evaluation study was a repeated measures, or within-subjects, controlled experiment comparing working face-to-face and working remotely, with the order of conditions counterbalanced. Twenty pairs of study participants (upper level undergraduate natural science students) conducted two realistic scientific research activities each requiring 2 to 3 hours to complete. Ten pairs of study participants worked face-to-face first and, on a different day, worked remotely (in different locations). Another ten pairs worked remotely first and, on a different day, face-to-face. When face-to-face, the participants shared a single collaboratory system; when collaborating remotely, each location was equipped with a complete collaboratory system.

The scientific research activities completed by the participants were designed in collaboration with natural scientists. The tasks were activities the scientists actually completed and documented during the course of their investigations. To complete the tasks the participants had to engage in the following activities typical of scientific research: operate the scientific equipment properly; capture and record data in their (electronic) notebook; perform analysis using scientific data analysis software applications and include the results of that analysis in their notebooks; draw conclusions, create hypotheses and support those hypotheses based on their data and analysis; and prepare a formal report of their work.

Task performance was measured through graded lab reports. The information participants were asked to provide in the reports mirrored the information scientists record in their lab notebooks. Each pair of study participants collaboratively created a lab report under each condition, generating a total of 40 lab reports; 20 created working remotely and 20 created working face-to-face. In addition, each participant was interviewed after each session. The post-interviews focused on participant's perceptions of the collaboratory system and their work patterns. 
The lab reports were graded blindly; the graders had no knowledge of the report's authors or under which condition the report was created. Initially one instructor created a grading template in collaboration with a physics professor. This instructor and two additional instructors used the template to grade a subset of lab reports (6). The instructors discussed differences among their grades and modified the grading template, adding further details, to reflect their discussion. An additional subset of reports (6) was graded using the updated template. Intercoder reliability was calculated for these assigned grades using Cohen's Kappa (Robson, 1993). Values of .75 and .79 were calculated for graded lab reports from the first and second task sessions respectively. Values above .70 are considered excellent (Robson, 1993). One instructor used the final template to grade all remaining lab reports.

The post-interviews were analyzed using both open coding and axial coding (Berg, 1989). During open coding a subset of the interviews were read thoroughly and carefully and coding categories, or coding frames, were identified. After the initial set of categories was discussed among the research team, three team members analyzed a subset of interviews. Additional coding categories emerged from this analysis. All three researchers analyzed an additional subset of interviews. No new coding categories emerged, and researchers were in agreement regarding the application of the codes. Intercoder reliability, calculated using Cohen's Kappa, yielded values of .86 and .81. During axial coding, the final step, all interviews were re-read and analyzed using the coding categories. Additional details regarding the experiment can be found in Sonnenwald, Whitton and Maglaughlin (2003).

\section{Situation Awareness Design Framework}

When individuals collaborate face-to-face, they share much, if not all, of the same physical environment and technology. Although each individual has different prior knowledge, the shared physical environment provides common points of reference that aid in communication and the development of shared meaning (Clark, 1996). That is, even though individuals' prior knowledge and abilities differ, the shared physical environment and ability to act and sense in that environment with some degree of control assists individuals in developing a shared and working situation awareness. When individuals collaborate across distances, not only do they have different prior knowledge and abilities, but also their physical environments are different. Each individual's situation awareness, including awareness of the local and remote situations, is mediated by technology.

How can technology support the development and maintenance of a shared situation awareness in the absence of a shared physical environment? In particular, what are the requirements for a system that needs to capture, or gather, information at one location of a distributed collaboration and deliver and display that information at one or more remote locations to support a shared situation awareness among participants at all locations? To address this question, we first examined what types of knowledge/information and knowledge individuals may need to develop and maintain a shared situation awareness. Next we examined how technology can support the acquisition and sharing of this information. 


\section{Information to Develop and Maintain Situation Awareness}

The literature and our empirical data suggest that situation awareness in scientific research collaboration requires several types of information, including contextual, task and process, and socio-emotional information (Figure 1). Distinguishing between these types of information facilitates our understanding of situation awareness and system requirements to support situation awareness.

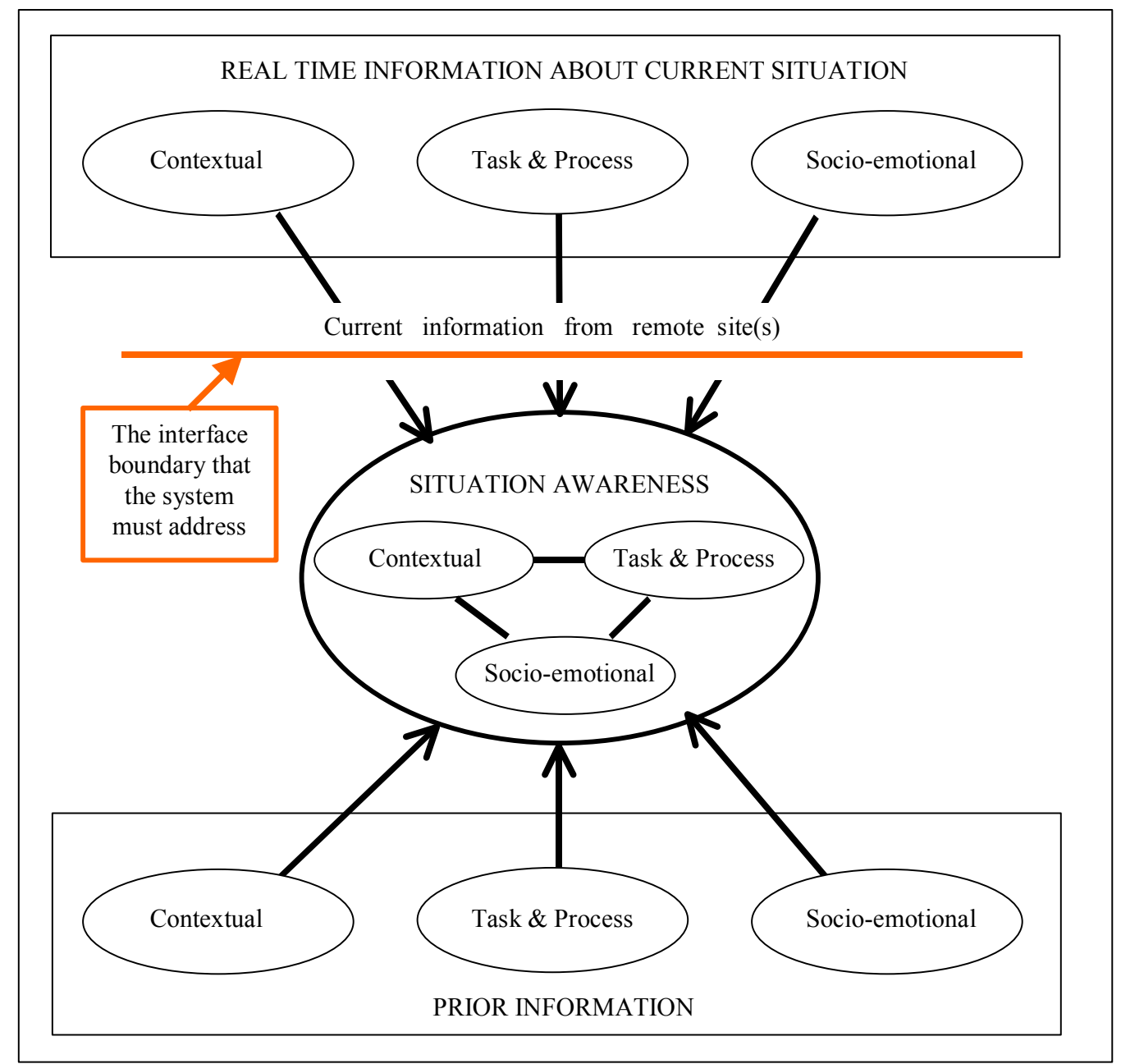

Figure 1. Current and prior contextual, task and process, and socio-emotional information are needed for situation awareness

Contextual information is a broad sense of the context in which things are happening. Context can be defined as a 'framework of meaning' (Cool, 2001, p. 8) or a 'framework of understanding' (Klein, 2000, p. 52.) Contextual information includes information regarding norms of scientific practice, research goals, organizational culture and work environment. Contextual information can vary between collaborators, as one scientist described: 
The person that we were collaborating with was so much into 'let's hurry up and publish this before so-and-so beats us and we won't get credit if they beat us.' ...And in the end, we found out the results weren't reproducible. I resisted all of the ideas this collaborator had to publish and in the end, it was the best thing I ever did because if we had published it we would have been wrong.

In a sense, contextual information includes the "rules of the game" and the "players in the game," and how to apply the rules. A scientist stated:

I don't mind the political games necessary to see a few things come together...To get an idea ...going...you have to get the blessings of various people.

When collaborators come from similar contexts, they may already know most of the contextual information relevant to the situation, reducing the amount of contextual information that must be mediated by technology. However, when collaborators come from different contexts, they need to be able to discover differences and similarities in their understanding of the context, and possibly discuss or negotiate those differences.

Task and process information is defined as information about current and relevant task activities and work processes. It includes information about tasks currently being performed and who is performing them. It also includes information about what tasks should be performed, how they can be performed, who can perform them and where and when they can be performed. ${ }^{2}$ Task and process information assists an individual in understanding what collaborators are doing. It also assists in creating expectations regarding what collaborators might do. When two collaborators share an in-depth understanding of processes, they may appear to function as one with work responsibilities passing smoothly between them.

An individual may increase his or her task and process information by observing the sequence of tasks another person or group of people are performing and by discussing tasks and processes with them. As one scientist explained:

Every now and then, she would look at us over the shoulder, I guess, and see how the experiment was going. And we talked with her too, every now and then.

Collaborators may have different task and process information, especially when collaborators come from difference disciplines or have different expertise. For example, a scientist told us:

\footnotetext{
${ }^{2}$ Embedded within task and process information is information about systems and other tools used during the scientific process. A large body of literature in the field of human-computer interaction provides design heuristics and methods to create system interfaces to effectively provide knowledge about the system. Thus, designing to facilitate the acquisition and use of system information is not covered in this paper.
} 
[My collaborators] will generate data and then they'll go 'we're going to run this through our computer software to see blah, blah, blah.'...I have no sense of what that involves. Is that a week of running a mainframe or is this something you put on there and click it and it comes back and says here's your picture?

Socio-emotional information is interpersonal information about collaborators. It includes information about their skills, work styles, approach to science, likes and dislikes, personality and emotional state. Several scientists discussed the important role socio-emotional information plays in their collaborative work:

Someone else's enthusiasm drives my interest in wanting to find the answer. A lot of people come in and say I want to do $X$ and $Y$ and then based on their commitment, you kind of say, well, he's not really interested so I'm not going to, you know, bend over backwards.

The best collaborations I have are the ones where the person I'm collaborating with thinks differently than I do...[this] is much more important than just getting experiments done more quickly.

The really wonderful thing is [that my collaborator] is a lot smarter than I am...collaborations are better... when I'm working with someone who I can learn a lot from in addition to bringing something to the table myself.

Interviewer: How do you judge whether somebody would be a good collaborator? What criteria do you use?

Scientist: What kind of behavior they have towards other people. Do they behave ethically? Are they forthright? Do they openly discuss their research or are they secretive? Are they, it may sound silly, but do I like them? ... do I find them interesting people with sort of the same kind of values that I have towards the science?

Bales (1950) and Nardi, Whittaker and Bradner (2000) have shown that groups, working both face-to-face and remotely, communicate socio-emotional information. They show tension, tension release, antagonism, enthusiasm, solidarity, agreement, disagreement and empathy through a variety of mechanisms including jokes, questions, assertions and body language.

Contextual, process and task, and socio-emotional information can be interrelated. Information or a lack of information of one type can enhance or limit one's understanding of other information. For example, a scientist described collaborating with a professional and not understanding why the professional did not complete several tasks. The scientist lacked contextual and socio-emotional information about his collaborator, and could not understand the task and process information at hand: 
[He] is a clinician that deals with children who have this lethal disease...I just cannot seem to ever get him to come over or respond to e-mails...[is] he so inundated with clinical stuff that he can't carve out of his day what he needs to do the scientific? ...I don't understand that...He can treat these patients for his whole career. Here's an opportunity to potentially bring a cure to them, and I don't understand why [he] can't say this is a priority.

This lack of contextual and socio-emotional information not only hindered the immediate collaboration but also future collaborations.

In summary, we propose situation awareness is built on a foundation of contextual, task and process, and socio-emotional information from previous situations. Current situation awareness is a synthesis of both current and past information. Poorly designed collaboration systems not only reduce the quality of current work but also of future work. This underscores the need for well-designed collaboration systems.

\section{Technology Features to Enable Situation Awareness}

As discussed above, when scientists collaborate face-to-face, they share an immediate environment and can develop situation awareness using contextual, process and task information gained through exploring, or experiencing, the (local) environment independently and/or collaboratively. However, when collaborating across distances, this exploration must occur across multiple environments (mine and yours). The exploration of the remote environments is no longer a direct experience, but is mediated by technology. It is important to design systems that enable scientists to obtain contextual, task and process, and socio-emotional information about the remote environments independently and/or collaboratively.

Substituting "virtual" for "remote" in the previous sentence makes obvious a parallel between collaboration technology and virtual reality (VR) technology. In collaboration technology, the goal is to enable users to create and maintain situation awareness at the remote and local sites; in virtual reality technology, the goal is for users to create and maintain a sense of presence, or "being there" in a place other than where she is physically. Our observation of this similarity led us to review research on requirements for VR systems.

Virtual reality research suggests several attributes, or factors, of virtual reality systems contribute to providing a sense of presence (Held \& Dulach, 1992; Sheridan, 1992; Witmer $\&$ Singer, 1992, 1996). More recent research on the effectiveness of virtual reality systems (e.g., Basdogan, Ho, Srinivasan, \& Slater, 2000; Usoh, Arthur, Whitton, Bastos, Steed, Slater, \& Brooks, 1999) has investigated the impact of a specific system attribute on VR effectiveness. The collection of system attributes proposed by Witmer and Singer (1992) remains useful and we employ it here. 
Table 1

Design Framework

Technology Attributes

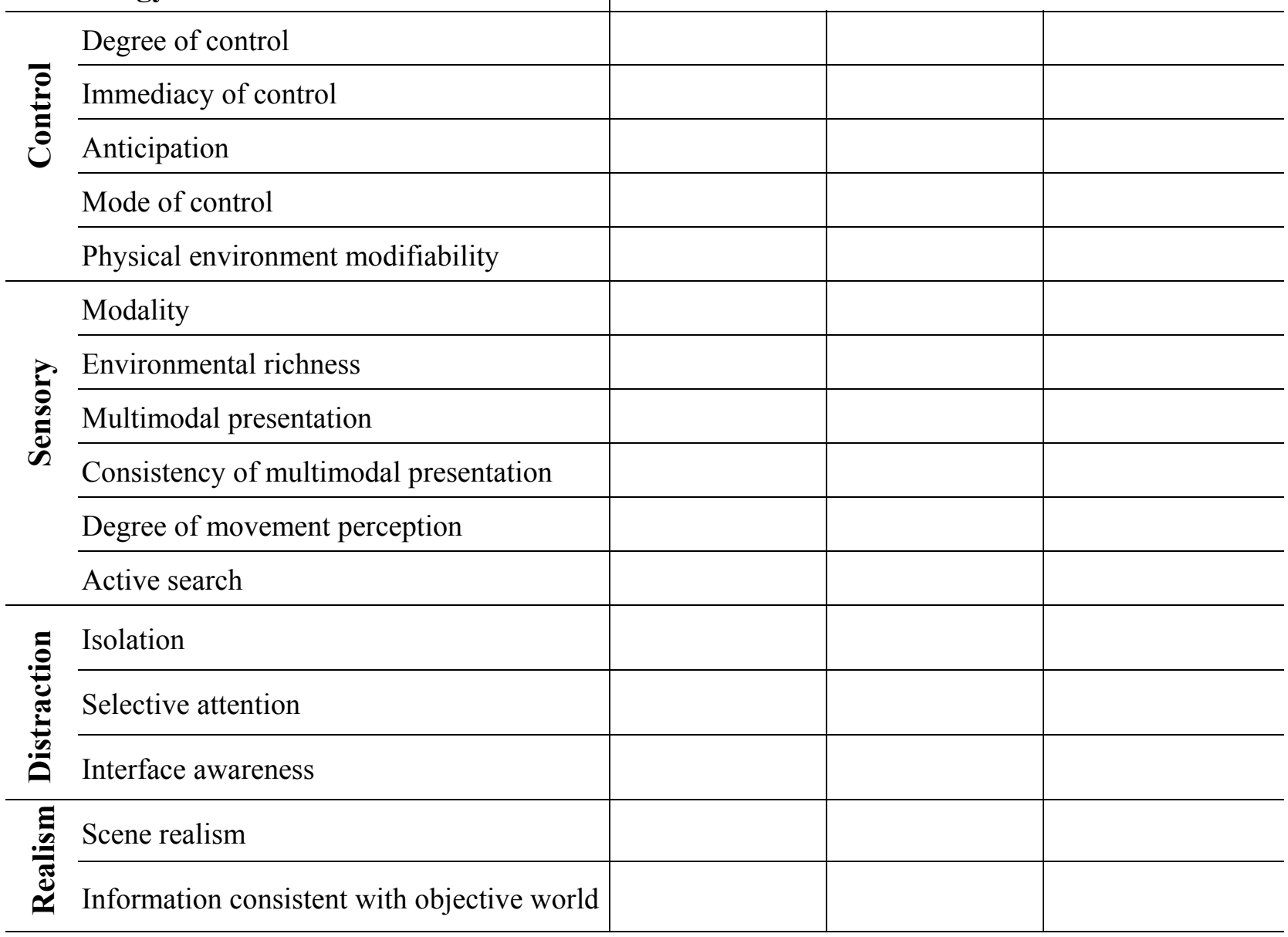

Witmer and Singer organize VR system attributes into four groups: control, sensory, distraction, and realism. Control attributes describe how well the user can interact with and change the virtual or remote environment. Sensory attributes are concerned with delivering information about the remote environment to the remote user, allowing the user to move through the remote environment and to actively and purposefully explore it. Just as systems must provide appropriate sensory stimuli, they must also minimize irrelevant external stimuli, or distraction attributes, that are not a part of, and particularly are inconsistent with, the stimuli from a remote environment. Realism attributes concern how much the remote world is like the natural world, i.e., the degree of consistency between the users' experience of the real world and their experience of the remote place. ${ }^{3}$

\footnotetext{
${ }^{3}$ Two of Witmer and Singer's realism attributes, meaningfulness of experience and separation anxiety/ disorientation, are not dependent upon the system technology, but the application domain. We omit these factors from our framework.
} 
We propose that, when designing a collaboration system, these attributes should be considered with respect to their ability to facilitate access to contextual task and process, and socio-emotional information. A table, with each row representing an attribute and each column representing a type of information, can be created to assist in this process (Table 1). Each blank cell in the table represents something to consider during the design process.

\section{Applying the Framework}

We applied the framework when designing the nanoManipulator collaboratory. Following is a discussion of the technology attributes, and their importance to contextual, task and process, and socio-emotional information. Examples of design decisions that we made using the framework are provided. However, due to space limitations not all design decisions made based on the framework are presented here.

\section{Control Attributes}

Degree of control. Degree of control refers to the number of elements in the remote and local environments that the user can control and the extent of that control. The more control that collaborators have over the remote environment, the greater their situation awareness. The more control that collaborators have over the local environment, the easier it is to proactively provide contextual, task and process, or socio-emotional information to a remote collaborator. For example, controlling the movement of specific objects in the remote environment, such as a microscope tip, enhances a collaborator's task and process information. Controlling the focus of a local camera on task activities can help increase a remote collaborator's task and process information. The capability to reserve a system for an experiment and to learn about what experiments are planned will increase contextual information. Thus, we designed the system with these features.

Immediacy of control. Immediacy of control focuses on system responsiveness. The smaller the delay between initiating system function (in the local or remote environment) and seeing its impact, the greater the sense of presence that is afforded. This enhances situation awareness by providing the means by which scientists can confirm actions. Software mechanisms that provide feedback as well as efficient algorithms and high network transmission speeds with low latency are typically needed to support this feature.

Anticipation. Anticipation is supported through media richness and consistency. When scientists conduct experiments while working face-to-face, they can recognize the activities being done and the status of those activities, and anticipate subsequent activities. For example, they can gather socio-emotional information, such as frustration and excitement, and anticipate responses, such as encouragement. We addressed this by providing multiple (high resolution and low latency) video camera views of the remote scientists.

Mode of control. In a collaborative system, a person may need to perform an action in one environment in order to cause a responding action in the other environment. Situation awareness is facilitated when that action is natural and similar across environments. For 
example, actions to change parameters for a data visualization should be exactly the same at all locations.

Physical environment modifiability. Situation awareness is enhanced when collaborators are able to modify the same artifacts in the remote environment as they could if collocated. For the nM system, this includes actions such as pushing, touching and/or modifying a sample during an experiment. In the stand-alone nM system, a scientist could perform these actions locally. We designed the collaboratory nM system to enable a scientist to perform the same actions when working remotely.

\section{Sensory Attributes}

Sensory modality. This attribute implies that systems should avoid forcing users to substitute one sensory mode for another. For example, although audio communication is closely related to collaborative task accomplishment, visual information is a strong source of socio-emotional, task and contextual knowledge (Bly, Harrison, \& Irwin, 1993; Monk \& Watts 1995; Daly Jones, Monk, \&Watts; 1998), and its absence in a collaboration system would diminish the effectiveness of the system. In the nM system, haptic feedback is also provided when the microscope tip is pushed against a sample.

The design framework suggests that providing haptic sensory feedback to users when their (remote) collaborator pushes the haptic device, allowing "you can feel what I feel", would be an efficient way to provide task and process information regarding haptic device manipulation. However, due to resource and technology constraints this feature was not implemented. The sensory modality attribute was disregarded in this instance; the remote collaborator can only provide visual and audio guidance with respect to haptic device manipulation. The framework cannot eliminate conflicts between system requirements and development costs, but rather illuminates possibilities and their potential importance.

Environmental richness. Environmental richness implies that systems should gather and display a variety of contextual, task and process, and socio-emotional information at adequate resolution and update rates. This implies a need for high quality video connections that show facial expressions, gestures and local objects; high quality audio connections; and shared applications to increase the richness of the environment. In addition, a "window within a window" to view a collaborator's remote screen while still viewing your local screen may enhance contextual and process knowledge.

Multimodal presentation. When the senses of sight, hearing, smell and touch are stimulated in an integrated and complete manner, situation awareness may be increased. Our observations of scientists engaged in audio-video conferences reveal that the more artifacts brought into the discussion (such as shared drawing tools or shared documents), the more the participants become engaged in the discussions. In a collaborative virtual environment, such senses may include touch integrated with sight and sound. In the $\mathrm{nM}$ collaboratory system, haptic feedback is integrated with visual information coming from the visualization screen, contributing task and process knowledge. 
Consistency of multimodal presentation. When visual, audio and haptic information are consistent and synchronized, people can more easily understand information. This can increase their confidence in, and use of, the information. Time synchrony across data presentation modes is an important component of consistent multimodal presentation. Network transmission times and latency can vary according to network loads and conditions. We, as have others, decided to send data and video over an Internet 2 network and audio over the public telephone network, distributing the network load and increasing redundancy to facilitate problem solving should network interruptions occur.

Degree of movement perception. This attribute focuses on self-motion within an environment. Zahorik and Jenison (1998) believe that presence is enhanced when one understands the result of an action in an environment, whether that environment is virtual or real. Ideally, a collaborator should be able to clearly see and hear actions that occur in the remote location as a result of a local action.

Active search. Active search capabilities allow users to control sensors at remote locations to obtain desired information. When collaborators can modify sensors to effectively search the remote environment, their socio-emotional, task and process, and contextual information can increase. The scientists we interviewed indicated that they would like to have multiple, pre-set video views of a remote collaborator's environment and the ability to modify those views dynamically using remotely controlled pan-tiltzoom camera mounts and/or automatic tracking cameras. Previous research (e.g., Bellotti \& Dourish, 1997; Harrison, Bly, \& Anderson, 1997) has also illustrated the importance of providing the ability to switch between multiple camera views, as well as repositioning and refocusing cameras.

\section{Distraction Attributes}

Isolation. Isolation refers to the extent that the user is physically shielded from nonrelevant, or distracting, information or activities in the local and remote environments. For example, devices that isolate a user from non-relevant aspects of the local environment can enhance his or her ability to gather and understand information from a remote environment. An example is the use of headphones to reduce ambient noise in the local environment so that a user may fully concentrate on the interaction with a remote collaborator. However, our observations of scientists using desktop audio-video conferencing tools such as NetMeeting ${ }^{\mathrm{TM}}$ also show that they like to hear auxiliary conversations arising in the local and remote environment to increase their contextual situation awareness. A solution is to provide options regarding audio headsets and audio speakers.

Selective attention. This attribute focuses on the extent users ignore non-relevant information. For example, a collaborator's willingness to ignore distractions in the local environment should enhance their awareness of the remote environment. This is a psychological issue. When observing collaborative meetings using only a speakerphone versus meetings using audio-video conferencing tools, we found that in the speakerphone situation, participants became distracted, looking around themselves. In the audio-video conferencing situation, participants paid closer attention to the discussion because (as they 
reported) they had eye contact with the distant collaborator. This is another reason why we included video-conferencing in our system.

Another method to capture and focus the attention of another person, particularly with respect to information on a monitor, is through pointing. We frequently observed scientists pointing to computer screens with their mouse pointer, fingers and pens to selectively focus a collaborator's attention. Thus, we designed our system to enable each user's pointer to be viewable by all collaborators.

Interface awareness. This attribute focuses on human-computer interface design. The human-computer interface for all types of information should be natural and easy to use. This has been previously proposed for all types of systems, and widely discussed in the human computer interaction literature (e.g., Shneiderman, 1998).

\section{Realism Attributes}

Scene realism. Scene realism, or the realistic rendering of the remote environment, addresses the validity of information from the remote environment used to develop situation awareness. Scene realism can be developed using real world content, e.g., video, and simulated content, e.g., computer animation or graphical representations. It is affected by camera resolution, light sources, field of view, as well as the connectedness and continuity of information being presented (Witmer \& Singer, 1998). Emerging technology, such as 3-D video conferencing (Towles, Chen, Yang, Kum, Fuchs, Kelshikar, Mulligan, Daniilidis, Holding, Zeleznik, Sadagic, \& Lanier, submitted), has the potential to increase scene realism.

Consistency of information with the natural world. Information about the remote environment provided by the system should be consistent with information learned through first-hand experiences. For example, if a scientist had previously visited a collaborator's lab then information about the lab, e.g., a floor plan, provided by the system should be consistent with the scientist's existing knowledge of the lab. Even when scientists have not had the opportunity to visit a collaborator's environment, they have expectations regarding that environment based on their previous experiences. Information provided by the system should be consistent with these expectations. For example, we originally designed the user interface of a collaborative tool using a space metaphor, with different types of rooms representing different system functionality. However, the scientists' experience with space in the natural world was different; in their world space was not partitioned by functionality. We changed the interface accordingly. 


\section{Evaluation of Task Performance}

Table 2

Graded Lab Report Statistics

\begin{tabular}{|c|c|c|c|c|c|c|c|c|c|c|}
\hline \multirow[b]{3}{*}{ Condition } & \multicolumn{10}{|c|}{ Graded Lab Report Scores (max. score $=100$ ) } \\
\hline & \multicolumn{5}{|c|}{ Collaborated FtF first $(\mathrm{n}=20)$} & \multicolumn{5}{|c|}{ Collaborated remotely first $(\mathrm{n}=20)$} \\
\hline & Mean & $\mathrm{SD}$ & Max & Min & Range & Mean & $\mathrm{SD}$ & Max & Min & Range \\
\hline Face-to-Face & 70.0 & 16.75 & 88 & 42 & 46 & 86.4 & 10.52 & 98 & 70 & 28 \\
\hline Remote & 75.1 & 10.49 & 89 & 56 & 33 & 70.0 & 8.89 & 80 & 55 & 25 \\
\hline
\end{tabular}

To investigate the validity and utility of the framework, we can evaluate the systems it helps to create. This is an indirect measure, and conclusions from the evaluation should be interpreted with caution.

As discussed earlier, we evaluated the collaboratory system using a repeated measures controlled experiment. Our hypotheses followed previous research (e.g., Olson \& Olson, 2000) that would predict that task performance and perceptions of the system when using it to collaborate across distances would be lower because the remote sessions would lack the richness of collocation and face-to-face interaction, including multiple and redundant communication channels, implicit cues, and spatial co-references. This lack of richness is often thought to impair situation awareness and subsequently have a negative impact on task performance and perceptions of technology.

In the experimental evaluation, task performance was measured by graded lab reports. The average lab report scores for the first task session were identical (70/100) for both the face-to-face and remote condition (Table 2). Although a null result statistically, the comparable scores between the two conditions on the first task are encouraging.

Data analysis further indicated that in this study collaborating remotely first had a positive effect on the second, face-to-face interaction. Using a multivariate analysis of variance (MANOVA) test, the differences in scores for the face-to-face and remote conditions were not statistically significant at the $p<=0.05$ level. ${ }^{4}$ However, when order is taken into account, participants who collaborated remotely first scored significantly higher on task 2 than did those who collaborated face-to-face first $(\mathrm{df}=1, \mathrm{~F}=9.66, p<0.01)$. This somewhat surprising result should be interpreted with caution. Due to available resources, we did not study the cases where participants completed the two task sessions under the same condition, e.g., both face-to-face or both remotely, and thus we are unable to eliminate the possible effect of task differences between the two sessions.

\footnotetext{
${ }^{4}$ The average lab report scores were greater in the second task session for both conditions, indicating a possible learning effect. This difference is accounted for in the analysis of variance computation.
} 
Participants' perceptions regarding control, sensory, distraction and realism attributes of the collaboratory system emerged from the analysis of the interview data. Participants requested several features the framework predicted would be important but were not implemented due to technical constraints, reported negative perceptions of features that did not conform to the framework, and reported positive regarding features the framework predicted would be important and which were implemented.

An example of features suggested by the framework and not implemented are automatic tracking or remotely controlled pan-tilt-zoom camera capabilities. These features were originally suggested when considering the active search (sensory) attribute. In postinterviews, several participants requested these features:

We didn't want to waste our time always adjusting the camera...have the camera follow you.

Interviewer/Observer: When you changed your seat [to work on a different part of the system], you changed the camera [view]...

Participant: Yeah, I would like to be able to see [my partner's face][all] the time.

Similarly, a participant requested the capability to view a collaborator's remote screen while viewing their local screen, a feature suggested by the environmental richness (sensory) attribute. The participant explained:

It would be good...if you're both in your private state [if] you could each see what the other's doing... if you have two different ideas of how to go about something, then you each can try it and see if you get to the same point without having to flip back and forth between [states.]

Study participants also reported negative perceptions of features that did not conform to the framework. For example, the consistency of multimodal presentation (sensory) attribute emerged as problematic for study participants. In particular, the video would "freeze" and be out of sync with the audio. Participants commented:

The video window froze and that was slightly aggravating.

[The video] kept stopping...his picture would freeze...the audio would be far ahead of where the video was.

Other participants commented:

[The video] was extremely helpful...I couldn't really describe [a scientific phenomenon] as well as I could just move my hands... in front of the camera.

I liked the video conferencing...I like seeing people as I interact with them and they react. 
Thus the video had utility, but the multimodal presentation of the video and audio was not effective. This particular problem can be addressed through improvements in networking infrastructure and algorithms that provide faster and more reliable video transmission and coding and decoding. These types of issues are typically outside the scope of software applications, yet they impact users' perceptions of an application.

Participants also reported positive perceptions of features that were suggested by the framework and implemented. This was particularly evident with respect to the mode of control attribute. In the nanoManipulator visualization software component, all users can execute system functions concurrently. Thus the model of control is identical when working individually and collaboratively. Participants commented:

The best thing was...the ability to work on the same thing at the same time with the nanoManipulator.

[We] never fought over the nanoManipulator because...both of us [could] use it at the same time.]

In comparison, the mode of control differed when working individually and collaboratively in the off-the-shelf shared application software that we used. For example, in NetMeeting ${ }^{\mathrm{TM}}$ users were required to explicitly take control of a shared application by double-clicking on the application window. One participant explained:

[It] became exceedingly frustrating... to share control... When I wanted to do something and my partner wanted to do something at the same time, we ...went back and forth double-clicking to gain control, and...it took us a few seconds to even acknowledge that. Essentially... we were fighting over control.

Task performance as measured by graded lab reports and perceptions of the system as discussed in post-interviews help demonstrate the appropriateness of the features suggested by the framework and provide some insights regarding the validity and utility of the framework. Yet these results should be interpreted with caution because the evaluation data was collected using a controlled experimental setting (as compared with a longitudinal, ethnographic study) and indirect measures of validity and utility were used.

\section{Limitations}

It may not be possible or necessary for a system to equally support the acquisition and dissemination of contextual, task and process, and socio-emotional information. Emphasizing one or two types of information may help prioritize design and implementation decisions. For example, we determined that our application would benefit from an increased emphasis on task and process information. This determination was made after analyzing the scientific context of the nM (Sonnenwald, et al, 2001). This emphasis guided our selection of design and implementation alternatives. The framework presented in this paper identifies types of information to support situation awareness but it does not prioritize them. Future research is needed to investigate guidelines for prioritization. 
Furthermore, we do not know if the list of attributes in the framework is exhaustive. Additional categories of attributes and additional attributes within categories may emerge as technology and our understanding of human information processing evolves.

We found that due to technical and budget constraints, some design decisions suggested by the framework could not be implemented. For example, we were not able to implement a "picture in a picture" feature that allows collaborators to see both their scientific visualization and their partner's visualization simultaneously, increasing the environmental richness. It is not unusual in design and development projects that design decisions are tempered by economic and political constraints. The framework does not solve this dilemma; it merely guides or helps inform decisions and solutions from a situation awareness perspective.

Lastly, exploring the applicability of the framework in other domains would increase our understanding of the strengths and weaknesses of the framework.

\section{Conclusions}

Scientific research is a complex, dynamic process in which situation awareness plays an important role. In scientific collaboration across distances, situation awareness is mediated by technology. Understanding how to design systems that augment and enhance scientists' situation awareness during the research process is a challenge.

To address this challenge, we build on previous research in situation awareness as well as interviews and observations of scientists to illuminate the complexity of situation awareness in scientific research and to propose that contextual, task and process, and socio-emotional information is needed to create and maintain situation awareness. Research in virtual reality systems suggests control, sensory, distraction and realism attributes of technology contribute to a sense of presence. We suggest that consideration of these attributes with respect to contextual, task and process, and socio-emotional information provides insights to guide design decisions.

We utilized the framework to guide decisions regarding technology to support situation awareness when designing the $\mathrm{nM}$ collaboratory system. As a result, the $\mathrm{nM}$ collaboratory system includes: consistent shared and private work modes, or spaces; the ability to dynamically switch between those shared and private work modes; the ability to customize an individual view of a shared work space; and multiple pointers that indicate each collaborator's focus of attention, interaction mode and actions simultaneously to all remote sites when in shared mode. Additional elements include a telephone for audio communication and video conferencing to provide views of hand gestures and other physical objects scientists use during experiments in addition to facial views of collaborators.

Previous studies have also illustrated the importance of several of these system features. This strengthens the face validity of the framework and highlights its potential to support design across a variety of application domains. 
We conducted a repeated measures, or within-subjects, controlled experimental evaluation of the collaboratory system that we designed using the framework. The experiment compared working face-to-face and working remotely with the order of conditions counterbalanced. Task performance was measured by graded lab reports. Average lab scores for participant pairs working remotely and face-to-face were identical for the first task, and study participants who worked remotely first had slightly improved performance when subsequently working face-to-face. Analysis of post-interview data revealed that participants requested several features the framework predicted would be important but were not implemented, reported negative perceptions of features that did not conform to the framework, and reported positive regarding features the framework predicted would be important and which were implemented. These results help illustrate the validity and utility of the framework, yet should be interpreted with caution because they are indirect measures of validity and utility.

In addition to providing design guidance, the framework may also guide the evaluation of technology that strives to support situation awareness. A survey instrument and/or interview questions could be developed based on the framework. Of course, additional studies that explored the applicability of the framework in a variety of domains are needed to further illustrate the strengths and weaknesses of the framework.

\section{Acknowledgements}

Our thanks to the study participants; to the team who have and continue to build the nanoManipulator collaboratory system, including Frederick P. Brooks, Jr., Martin Guthold, Aron Helser, Tom Hudson, Kevin Jeffay, David Marshburn, Don Smith, Richard Superfine, and Russell M. Taylor II; and thanks to Ron Bergquist, Bin Li, Atsuko Negishi, Leila Plummer and Eileen Kupsas-Soo who assisted during this research.

This research has been funded by the NIH National Center for Research Resources, NCRR 5-P41-RR02170, and the NIH National Institute of Biomedical Imaging and Bioengineering, P41-EB-002025. The nanoManipulator collaboratory project is part of the Center for Computer Integrated Systems for Microscopy and Manipulation at the University of North Carolina at Chapel Hill.

\section{References}

Bafoutsou, G., \& Mentzas, G. (2002). Review and functional classification of collaborative systems. International Journal of Information Management, 22, 281-303.

Bales, R. (1950). Interaction Protocol Analysis. Cambridge, MA: Addison-Wesley.

Basdogan, C., Ho, C., Srinivasan, M., \& Slater, M. (2000). An experimental study on the role of touch in shared virtual environments. ACM Transactions on Computer-Human Interaction, 7(4), 443-460. 
Bellotti, V., \& Dourish, P. (1997). Rant and RAVE: Experimental and experiential accounts of a media space. In K. Finn, A. Sellen, \& S. Wilbur (Eds.), Video-Mediated Communication (pp. 245-272). Mahwah, NJ: Lawrence Erlbaum.

Berg, B. L. (1989). Qualitative research methods for the social sciences. Boston: Allyn and Bacon.

Bloomberg, J., Giacomi, J. Mosher, A., \& Swenton-Hall, P. (1993). Ethnographic field methods and their relation to design. In: Schuler, D. \& Namioka, A. (Eds), Participatory Design: Principles and Practices (pp. 123-155). Hillsdale, NJ: Lawrence Erlbaum Associates, Inc.

Bloomberg, J., Suchman, L., \& Trigg, R. (1996). Reflections on a work-oriented design project. Human-Computer Interaction, 11, 237-265.

Bly, S.A., Harrison, S.R., \& Irwin, S. (1993). Media spaces: bringing people together in a video, audio, and computing environment. Communications of the ACM, 36, 28-48.

Cerf, V., Cameron, A., Lederberg, J., Russell, C., Schatz, B., Shames, P., Sproull, L., Weller, R., Wulf, W. (1993). National Collaboratories: Applying Information Technology for Scientific Research. Washington, DC: National Academy Press.

Cheverest, K., Mitchell, K., Davies, N., \& Smith, G. (2000). Exploiting context to support social awareness and social navigation. SIGGROUP Bulletin, 21(3), 43-48.

Clark, H. (1996). Using Language. Cambridge, UK: Cambridge University Press.

Cohen, D., Jacovi, M., Maarek, Y., \& Soroka, V. (2000). Collection awareness on the web via livemaps. SIGGROUP Bulletin, 21(3), 12-15.

Cool. C. (2001). The concept of situation in information science. In W. E. Williams (Ed.), Annual Review of Information Science and Technology, Vol. 35 (pp. 5-42). Medford, NJ: Information Today.

Daly Jones, O., Monk, A., \& Watts, L. (1998). Some advantages of video conferencing over high-quality audio conferencing: fluency and awareness of attentional focus. International Journal of Human Computer Studies, 49: 21-58.

Dominguez, C. (1994). Can SA be Defined? In M. Vidulich, C. Dominguez, E. Vogel, and G. McMillan (Eds.), Situation Awareness: Papers and annotated bibliography (pp. 5-15; Report AL/CF-TR-1994-0085). Wright-Patterson Airforce Base, OH: Air Force Systems Command. 
Dourish, P. \& Bly, S. (1992). Portholes: supporting awareness in a distributed work group. Proceedings of 1992 Conference on Human Factors in Computer Systems. (pp.541547). NY: ACM Press.

Endsley, M.R. (1995). Toward a Theory of Situation Awareness in Dynamic Systems. Human Factors, 37(1), 32-64.

Endsley, M.R. (2000). Theoretical underpinnings of situation awareness: A critical review. In M.R. Endsley \& D.J. Garland (Eds.), Situation Awareness Analysis and Measurement (pp. 3-32). Mahwah, NJ: Lawrence Erlbaum.

Finch, M., Chi, V., Taylor II, R.M., Falvo, M., et al. (1995). Surface modification tools in a virtual environment interface to a scanning probe microscope. Proceedings of the ACM Symposium on Interactive 3D Graphics - Special Issue of Computer Graphics. (pp. 1318). NY: ACM Press.

Finholt, T. (2001). Collaboratories. In B. Kronin (Ed.) Annual Review of Information Science and Technology (pp. 73-108). Washington, DC: American Society for Information Science and Technology.

Fish, R.S., Kraut, R.E., Root, R.W., \& Rice, R.E. (1993). Video as a technology for informal communication. Communication of the ACM, 36(1), 48-62.

Gutwin, C. \& Greenberg, S. (1997). Workspace Awareness. Position paper for the $A C M$ CHI'97 Workshop on Awareness in Collaborative Systems, organized by Susan E. McDaniel and Tom Brinck, Atlanta, Georgia, March 22-27.

Gutwin, C. \& Greenberg, S. (1998). Effects of Awareness Support on Groupware Usability. In Proceedings of the CHI'98 Conference on Human Factors in Computing Systems (pp.511-518). NY: ACM Press.

Gutwin, C. \& Greenberg, S. (1999). The Effects of Workspace Awareness Support on the Usability of Real-Time Distributed Groupware. ACM Transactions on Computer-Human Interaction, 6(3), 243-281.

Gutwin, C. \& Greenberg, S. (2000). The Mechanics of Collaboration: Developing Low Cost Usability Evaluation Methods for Shared Workspaces. IEEE 9th International Workshop on Enabling Technologies: Infrastructure for Collaborative Enterprises (WET-ICE'00). June 14-16. Gaithersburg, MD: NIST.

Gutwin, C. \& Greenberg, S. (2002). A descriptive framework of workspace awareness for real-time groupware. Computer Supported Cooperative Work, 11, 411-446.

Harrison, S., Bly, S., \& Anderson, S. (1997). The media space. In K. Finn, A. Sellen, \& S. Wilbur (Eds.), Video-Mediated Communication (pp. 273-300). Mahwah, NJ: Lawrence Erlbaum. 
Held, R., \& Durlach, H. (1992). Telepresence. Presence, 1(1), 109-112.

Huges, J.A., Randall, D., \& Shapiro, D. (1992). Faltering from ethnography to design. Proceedings of the Conference on Computer Supported Cooperative Work. ACM, New York, pp 115-122.

Kensing, F., Simonsen, J., \& Bodker, K. (1998). MUST: A method for participatory design. Human-Computer Interaction, 13, 167-198.

Klein, G. (2000). Analysis of situation awareness from critical incident reports. In M.R. Endsley \& D.J. Garland (Eds.), Situation Awareness Analysis and Measurement (pp. 5172). Mahwah, NJ: Lawrence Erlbaum.

Monk, A.F. \& Watts, L. (1995). Poor quality video links affects speech but not gaze. Proceedings of the CHI Conference (pp 274-275). NY: ACM Press.

Mumford, E. (1995). Effective Systems Design and Requirements Analysis: The ETHICS Approach. Basingstoke: Macmillan.

Murray, D. (1993). An ethnographic study of graphic designers. Proceedings of the Third European Conference on Computer-Supported Cooperative Work (pp 295-309.) NY: ACM Press.

Nardi, B., Whittaker, S., \& Bradner, E. (2000). Interaction and outeraction: Instant messaging in action. ACM 2000 Conference on Computer Supported Cooperative Work (pp. 79-88). NY: ACM Press.

Olson, G.M., \& Olson, J.S. (2000). Distance matters. Human-Computer Interaction, 15(2-3), 139-178.

Rasmussen, J., Pejtersen, A., \& Goodstein, L. (1994). Cognitive Systems Engineering. NY: Wiley.

Robson, C. (2002). Real World Research. Oxford, UK: Blackwell Publishers.

Rodden, T. (1996). Populating the application: A model of awareness for cooperative applications. In M. Ackerman (Ed.), Proceedings of the ACM 1996 Conference on Computer Supported Cooperative Work (pp. 87-96). NY: ACM Press.

Rutter, D.R., \& Stephenson, G.M. (1979). Role of visual communication in social interaction. Current Anthropology, 20, 124-125

Salas, E., Dickinson, T.L., Converse, S.A., \& Tannenbaum, S.I. (1992). Toward an Understanding of Team Performance and Training. In R.W. Swezey and E. Salas (Eds.). Teams: Their Training and Performance (pp. 3-29). Norwood, NJ: Ablex. 
Salas, E., Prince, C., Baker, D.P., \& Shresta, L. (1995). Situation awareness in team performance: Implications for measurement and training. Human Factors, 37(1), $123-$ 136.

Schmidt, K. (2002). The problem with 'awareness.' Computer Supported Cooperative Work, $11,285-298$.

Schutz, A., \& Luckmann, T. (1973). The Structures of the Life-World, Vol. I. Evanston, IL: Northwestern University Press.

Schutz, A., \& Luckmann, T. (1989). The Structures of the Life-World, Vol. II. Evanston, IL: Northwestern University Press.

Shneiderman, B. (1998). Designing the User Interface. Reading, MA: Addison Wesley.

Sheridan, T. (1992). Musings on telepresence and virtual presence. Presence, 1(1), 120-125.

Sohlenkamp, M. \& Chwelos, G. (1994). Integrating communication, cooperation and awareness: The DIVA virtual office environment. Proceedings of the Computer Supported Cooperative Work Conference (pp. 331-343.) Cambridge, MA: ACM Press.

Sonnenwald, D.H., Bergquist, R., Maglaughlin, K.L., Soo, E.K. \& Whitton, M. (2001). Designing to support scientific research across distances: The nanoManipulator environment. In E. Churchill, D. Snowdon \& A. Munro (Eds.). Collaborative Virtual Environments. London: Springer Verlag.

Sonnenwald, D.H., \& Pierce, L. G. (2000). Information behavior in dynamic group work contexts: Interwoven situation awareness, dense social networks and contested collaboration in command and control. Information Processing \& Management, 36, 461479.

Sonnenwald, D.H., Whitton, M.C., \& Maglaughlin, K.L. (2003). Evaluating a scientific collaboratory: Results of a controlled experiment. ACM Transactions on ComputerHuman Interaction (10)2, 150-176.

Suchman, L. (1995). Making work visible. Communications of the ACM, 38, 56-64.

Taylor II, R.M., \& Superfine, R. (1999). Advanced interfaces to scanning probe microscopes. In H.S. Nalwa (Ed.), Handbook of Nanostructured Materials, Vol. 10 (pp. 217-255). Norwood, NJ: Ablex.

Tysoe, M. (1984). Social cues and the negotiation process. British Journal of Social Psychology, 23, 61-67 
Tollmar, K., Sandor, O., \& Schömer, A. (1996). Supporting social awareness @ work: design and experience. Proceedings of the Computer Supported Cooperative Work Conference (pp. 298-306.) Cambridge, MA: ACM Press.

Towles, H., Chen, W., Yang, R., Kum, S., Fuchs, H., Kelshikar, N., Mulligan, J., Daniilidis, K., Holding, L., Zeleznik, B., Sadagic, A., \& Lanier, J. (submitted). 3D tele-immersion over internet2.

Usoh, M., Arthur, K., Whitton, M., Bastos, R., Steed, A., Slater, M., Brooks, F. (1999). Walking $>$ walking-in-place $>$ flying in virtual environments. In Proceedings of the $26^{\text {th }}$ Annual Conference on Computer Graphics and Interactive Techniques (pp. 359-364). NY: ACM Press.

Wellens, A.R. (1993). Group situation awareness and distributed decision making from military to civilian applications. In N.J. Castellan, Jr. (Ed.), Individual and Group Decisions Making: Current Issues (pp. 267-291). Hillsdale, NJ: Erlbaum.

Witmer, B.G. \& Singer, M.J. (1992). Measuring Presence in Virtual Environments (DTIC Reference Number AD A286 183 DTIC TR 1014): Ft. Belvoir, VA: DTIC, Defense Technical Information Center.

Witmer, B.G. \& Singer, M.J. (1998). Measuring presence in virtual environments: A presence questionnaire. Presence, 7, 225-240.

Wulf, W.A. (1989). The National Collaboratory: A White Paper. Appendix A in Toward a National Collaboratory, unpublished report of a National Science Foundation invitational workshop held at Rockefeller University.

Wulf, W.A. (1993). The collaboratory opportunity. Science, 261, 854-855.

Zahorik, P. \& Jenison, R.L. (1998). Presence as being-in-the-world. Presence, 7, 78-89. 